\title{
STUDY OF MAGNETOVISCOSITY AND SPIN VELOCITY OF A FERRFOFLUID FLOW BETWEEN INCLINED PLANES
}

\author{
C. S. Asha ${ }^{1} \&$ L. N. Achala ${ }^{2}$
}

Abstract-In this paper we study the flow of an incompressible viscous, ferrofluid through infinite parallel plates inclined at an angle $\theta$ with horizontal axis in presence of external magnetic field.Ferrofluid rheology depends on the external magnetic field applied. Ferrofluid rheology depends on the external magnetic field applied and pressure gradient. The presence of body couple plays very important role in ferrofluids. The behaviour of spin velocity is studied with and without body couple. It is observed that spin velocity obey a third order algebraic equation due to an imposed magnetic field $\vec{H}$. The effect of external magnetic field and pressure gradient on spin velocity are studied and explained through graphs. Effective viscosity effects on spin velocity are also studied. The plate inclination and pressure gradient are responsible for reverse flow in spin velocity.

Keywords - Magnetic field, Magnetization, Pressure gradient, Body couple, Spin velocity, gravitational force.

\section{INTRODUCTION}

Ferrofluids is a suspension of colloidal liquid stably dispersed in an appropriate carrier liquid. The interaction of magnetic moment of suspended particles with externally applied magnetic field yields two different basic effects. The magnetic field gradient yields a force on fluid whereas magnetic moment of particles will experience a torque which tries to align with magnetic field direction. Thus ferrofluids or magnetic liquids have unusual properties due to which they offer unique advantages with a wide range of applications for emerging technologies. Ferrofluids and their application was first identified by NASA Scientists at Lewis research centre for filling up fuel to the engine of an orbiting spacecraft [1, 2, 3]. At AVCO space system division, scientists used ferrofluid ring for temperature control of space craft which was very hot on the side facing the sun and very cold on the other side. Thus Dr. Ronald Moskowitz and Dr. Ronald Rosensweig, both from AVCO space systems division realized that ferrofluids offer wide application to vast problems. They found the use of ferrofluid in manufacturing of semiconductor chips for electronic system (magnetic seals). Ferrofluids are also used as performance improver for hi fi loud speakers. Ferrofluids have wider acceptance in a variety of industrial processes medical instruments, silicon crystal growths, plasma processes and so on. Ferrofluids can be used in medical applications, ferrofluid coated material is used to manufacture drugs. In that process they need to pass that material through two parallel belts wherein one will be fixed and other will be moving to carry the ferrofluid coated material. This problem can be modelled as a flow between two infinite parallel plates $[1,4,5,3]$.

Viscous incompressible fluid between two coaxial cylinders rotating about common axis is studied by many theoretically and experimentally $[6,7,8,9]$. Magnetic fluid flows and their detailed study was given by $[4,5,3]$. Influence of a magnetic field on the Taylor instability in magnetic field was studied by Vislovich [11], Niklas, Stiles, Odenbach, Chang, Harris [11, 12, 13, $14,15]$ have studied this problem extensively for different applied magnetic field. Felderhof [16] discussed entropy production and its relation to magnetoviscosity in both planar Couette flow and Poiseuille pipe flow in parallel and perpendicular magnetic field. McTague, Bacri et al., Zeuner et al., Kamiyama [17, 18, 19, 20] has experimentally studied Poiseuille flow. Marsenyuk [21] provided a prediction to experimental data of Bacri et al. [18]. Zahn and Pioch [22] have investigated the torque of ferrofluid particles for four different unsteady magnetic fields. Felderhof [5] studied Poiseuille flow with linear polarized oscillating magnetic field directed down the axis of pipe. Gazeau et al. [23] have studied energy conversion in ferrofluids with magnetic nano particles. Schumacher [24] experimentally studied the shear dependence of the ferrofluid viscosity and the pipe flow rate in a linearly polarized magnetic field oscillating along the axis of the pipe. Krekhov et al. [25] theoretically studied ferrofluid pipe flow in an oscillating magnetic field along the pipe axis in a wide range of the flow rate and observed that the ferrofluids exhibit non newtonian behavior due to field dependent viscosity which indicates an alteration of velocity profile which is closer to parabolic and the flow rate deviates from the prescribed value given by the poiseuille's formula . Jitender and Renu [26] studied Taylor-Couette flow for incompressible ferrofluid in an annular space between two coaxially rotating cylinder in the prensence of axial magnetic field and observed the delay in the onset of the Taylor-Couette instability due to perturbations in magnetic field in the gap between the cylinders. He et al. [27] have studied effective

\footnotetext{
${ }^{1}$ P.G. Department of Mathematics and Research Centre in Applied Mathematics, M.E.S College of Arts, Commerce and Science, 15th Cross, Malleswaram,Bangalore-560003.

${ }^{2}$ P.G. Department of Mathematics and Research Centre in Applied Mathematics, M.E.S College of Arts, Commerce and Science, 15th Cross, Malleswaram,Bangalore-560003.
} 
magnetoviscosity of Planar-Couette magnetic fluid flow with applied uniform dc magnetic field transverse to the duct axis and described the conditions for multi valued effective magnetoviscosity and spin velocity .

In this paper we study the flow of ferrofluid between two conveyor belts(treated as plates) inclined at an angle $\theta$ to the horizontal axis. The paper is organized as follows. Section II consists of the governing equations for incompressible ferrofluids. In section III we have given geometry of the flow and solution. In section IV the results and discussions are presented. Section $\mathrm{V}$ contains the graphs.

\section{GOVERNING EQUATIONS}

We consider ferrohydrodynamic equations such as Continuity equation, Linear momentum equation, Angular momentum equation, Magnetization equation with Maxwell's equation given by [3] are as follows

$\nabla \cdot \vec{v}=0$,

$\rho\left[\frac{\partial \vec{v}}{\partial t}+(\vec{v} \cdot \nabla) \vec{v}\right]=-\nabla p+\mu_{0}(\vec{M} \cdot \nabla) \vec{H}+(\eta+\zeta) \nabla^{2} \vec{v}+2 \zeta \nabla \times \vec{\omega}+\rho \vec{g}$,

$\rho \vec{I}\left[\frac{\partial \vec{\omega}}{\partial t}+\vec{v} \cdot \nabla \vec{\omega}\right]=\eta^{\prime} \nabla^{2} \omega+\mu_{0} \vec{M} \times \vec{H}+2 \zeta(\nabla \times \vec{v}-2 \vec{\omega})$,

$\frac{\partial \vec{M}}{\partial t}+\vec{v} \cdot \nabla \vec{M}=\vec{\omega} \times \vec{M}-\frac{1}{\tau}\left(\vec{M}-\vec{M}_{0}\right)$,

$\vec{M}_{0}=\chi_{0} \vec{H}$,

$\nabla \cdot \vec{B}=0$

$\nabla \times \vec{H}=0$,

$\vec{B}=\mu_{0}(\vec{H}+\vec{M})$,

where $\vec{v}$ is the translational velocity, $\vec{\omega}$ is the spin velocity, $\vec{H}$ is the applied magnetic field, $\vec{M}$ is the Magnetization, $\vec{M}_{0}$ is the equilibrium magnetization, $\vec{I}$ is the moment of inertia density, $\rho$ is the density, $\tau$ is the magnetization relaxation time, $\eta$ is the coefficient of shear viscosity, $\zeta$ is the coefficient of vortex viscosity, $\eta^{\prime}$ is the shear coefficient of spin viscosity, $\chi_{0}$ is a constant depending on temperature and composition of suspension called magnetic susceptibility, $p$ is the pressure, $g$ is the gravitation, $\mu_{0}$ is the magnetic permeability.

\section{MATHEMATICAL FORMULATION AND SOLUTION}

Consider an incompressible steady ferrofluid flow between two inclined parallel plates at an angle $\theta$ to the horizontal axis separated by a distance d as shown in Fig.1. The magnetic field $\vec{H}$ or the magnetic flux density $\vec{B}$ is applied along $x$ direction.

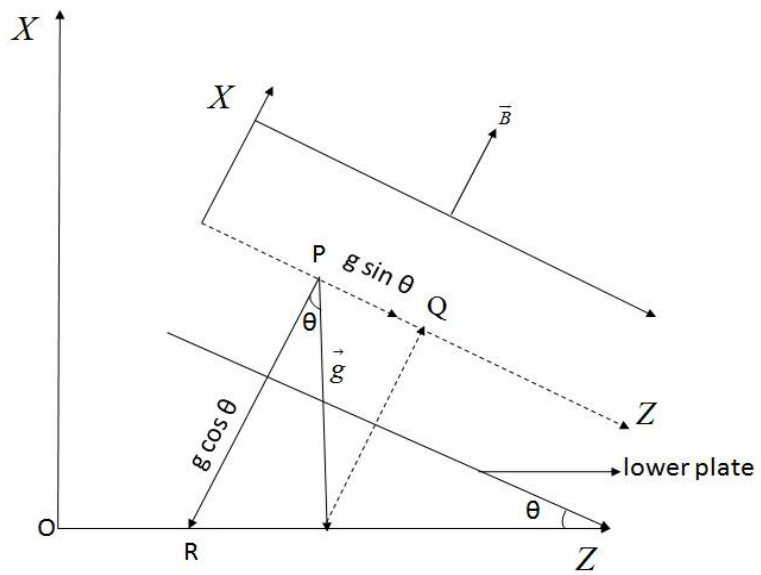

Figure 1: Geometry of Problem 
The fluid flow is in the $z$ - direction, therefore we have $v_{x}=0, v_{y}=0$ and also for small flow rates, the viscous forces avoid continuous acceleration of the fluid. Hence $v_{z}$ is independent of $z$. Therefore the velocity $v_{z}=v_{z}(x)$ and pressure $p=p(z)$.

The flow velocity $\vec{v}$ and the spin velocity $\vec{\omega}$ are considered as [27]

$\vec{v}=\left(0,0, v_{z}(x)\right), \vec{\omega}=\left(0, \omega_{y}(x), 0\right), \vec{g}=\left(g_{x}, 0, g_{z}\right)$.

Since the imposed magnetic field $\vec{H}$ or magnetic flux density $\vec{B}$ are spatially uniform with the y and z coordinate, all the field and flow variables are independent of $y$ and $z$ and can only vary with $x$.

$\vec{H}=\left(H_{x}, 0,0\right), \vec{B}=\left(B_{x}, 0, B_{z}(x)\right), \vec{M}=\left(M_{x}(x), 0, M_{z}(x)\right)$.

Using (10) in (6) and (3) we get,

$B_{x}=$ constant $H_{y}, H_{z}=$ constant $=0$.

Using (9) and (10) in (7) we obtain,

$$
\begin{aligned}
& M_{x}=\frac{\chi_{0} H_{x}}{\left[\left(\omega_{y} \tau\right)^{2}+1\right]}, \\
& M_{z}=\frac{-\chi_{0} H_{x} \omega_{y} \tau}{\left[\left(\omega_{y} \tau\right)^{2}+1\right]} .
\end{aligned}
$$

By neglecting spin viscosity and using (9),(10) and (12) the flow and spin velocity equations (2) and (6) reduces to

$$
\begin{gathered}
(\zeta+\eta) \frac{d^{2} v_{z}}{d x^{2}}+2 \zeta \frac{d \omega_{y}}{d x}-\frac{\partial p}{d z}+\rho g \sin \theta=0, \\
-\frac{\mu_{0} \chi_{0} H_{x}^{2} \tau \omega_{y}}{1+\left(\omega_{y} \tau\right)^{2}}-2 \zeta\left(\frac{d v_{z}}{d x}+2 \omega_{y}\right)=0 .
\end{gathered}
$$

\section{Case(i):}

When the magnetic material is soft the Magnetization $\vec{M}$ is parallel to the applied Magnetic field $\vec{H}$ then we can neglect body couple [27, 3]. Therefore for soft magnetic material the solution for flow and spin velocities by solving (13) and (14) with the boundary conditions

$v_{z}(x)=0$ at $x= \pm d$,

are

$$
\begin{aligned}
& v_{z}(x)=\frac{1}{2 \eta}\left[\rho g \sin \theta-\frac{\partial p}{\partial z}\right]\left(d^{2}-x^{2}\right), \\
& \omega_{y}(x)=\frac{1}{2 \eta}\left[\rho g \sin \theta-\frac{\partial p}{\partial z}\right] x .
\end{aligned}
$$

\section{Case(ii):}

When the magnetic material is hard it retains the magnetic moment after the applied magnetic field is removed. Hence we cannot neglect the body couple. Therefore for hard magnetic material solving (13) and (14) under the condition $\frac{d v_{z}(x)}{d x}=0$ at $x=0$ we get the spin velocity equation as

$\frac{\mu_{0} \chi_{0} H_{x}^{2} \tau \omega_{y}(x)}{4 \zeta\left(1+\left(\omega_{y}(x) \tau\right)^{2}\right)}+\frac{\zeta \omega_{y}(0)-\zeta \omega_{y}(x)-\frac{1}{2}\left(\rho g \sin \theta-\frac{\partial p}{\partial z}\right) x}{(\eta+\zeta)}+\omega_{y}(x)=0$.

(18) can be written as

$$
(1-A \zeta) \tau^{2} \omega_{y}^{3}(x)+\left(\zeta \omega_{y}(0)-P x\right) A \tau^{2} \omega_{y}^{2}(x)+\left(P_{H}+1-A \zeta\right) \omega_{y}(x)+\left(\zeta \omega_{y}(0)-P x\right) A=0,
$$


where

$A=\frac{1}{\eta+\zeta}, P=\frac{1}{2}\left(\rho g \sin \theta-\frac{\partial p}{\partial z}\right), P_{H}=\frac{\mu_{0} \chi_{0} H_{x}^{2} \tau}{4 \zeta}$

Solving (19) for real roots by taking $\omega_{y}(0)=0, x=1$, the graph is drawn for spin velocity $\omega_{y}$ versus $P_{H}=\frac{\mu_{0} \chi_{0} H_{x}^{2} \tau}{4 \zeta}$ for various values of $P=\frac{1}{2}\left[\rho g \sin \theta-\frac{\partial p}{\partial z}\right]$.

\section{Shear Stress:}

The shear stress is obtained as

$T_{z x}=(\eta+\zeta) \frac{d v_{z}(x)}{d x}+2 \zeta \omega_{y}(x) \Rightarrow T_{z x}=(\eta+\zeta)\left[\frac{-x}{\eta}\left(\rho g \sin \theta-\frac{\partial p}{\partial z}\right)\right]+2 \zeta \omega_{y}(x)$

In the absence of magnetic field that is $H_{x}=0 \Rightarrow \zeta=0$, the shear stress is given by

$T_{z x_{0}}=\eta \frac{d v_{z}(x)}{d x}=(-x)\left(\rho g \sin \theta-\frac{\partial p}{\partial z}\right)$

The effective shear stress is obtained as

$\Delta T_{z x}=T_{z x}-T_{z x_{0}}=\zeta\left[2 \omega_{y}(x)-\frac{x}{\eta}\left(\rho g \sin \theta-\frac{\partial p}{\partial z}\right)\right]$.

\section{Mass flow rate:}

The measurement of mass flow of a fluid is a important parameter in many processes. It is needed to know that the right fluid is at right place and in the right time. The mass flow is determined by measuring the fluid velocity. Velocity depends on the pressure differential which is forcing the fluid flow between plates and as the distance between plates is constant then the average velocity is an indication of flow rate. Mass flow rate can be used to identify flow separation also. The Mass flow rate is given by

$$
\begin{aligned}
& Q=\int_{-d}^{d} v_{z}(x) d x=\frac{2 d^{3}}{3 \eta}\left(\rho g \sin \theta-\frac{\partial p}{\partial z}\right) \\
& \text { At } x=0, v_{z}=v_{z_{\text {max }}}=\frac{d^{2}}{\eta} P, v_{z_{a v g}}=\frac{v_{z}}{2 d} .
\end{aligned}
$$

Therefore

$Q=\frac{2}{3} d v_{z_{\max }}$,

Or

$$
Q=\frac{1}{3} v_{z_{a v g}}
$$

\section{RESULTS AND DISCUSSIONS}

In this paper we study the flow of ferrofluid between infinite parallel plates inclined at an angle $\theta$ to the horizontal axis. The fluid considered is a strong ferrofluid. We have included both symmetric and antisymmetric shear stress. We discuss the dependence of magneto viscosity on spin velocity of the flow by varying total pressure in presence of body couple. The effect of total pressure on velocity is studied in the absence of body couple. It is observed in figure 2 that the velocity profile is parabolic. In figure 3 it is seen that spin velocity is also parabolic but due to the presence of body couple a reverse flow is seen. In figure 4, 5 and 6 it is observed that as $\theta$ increases the parabolic flow expands for various values of $\frac{\partial p}{\partial z}$. In figure 7,8, and 9 it is observed that when $\theta$ is less than $30^{\circ}$ the effect of pressure gradient is prominent. In figure 7 for $\frac{\partial p}{\partial z}=2.5$ the 
reverse flow of $\omega_{y}$ is observed for $\theta=\frac{\pi}{8}, \frac{\pi}{10}, \frac{\pi}{12}$ in positive direction whereas for $\theta=\frac{\pi}{14}$ it is in negative direction. For $\frac{\partial p}{\partial z}=3$ the flow of $\omega_{y}$ is reversed in negative direction for $\frac{\pi}{12}$ and $\frac{\pi}{14}$ (figure 8), similarly for $\frac{\partial p}{\partial z}=3.5$ the reverse flow in negative direction is seen for $\frac{\pi}{10}, \frac{\pi}{12}, \frac{\pi}{14}$ (figure 9). Thus we conclude that pressure plays very important role in controlling flow of such type.

\section{GRAPHS}

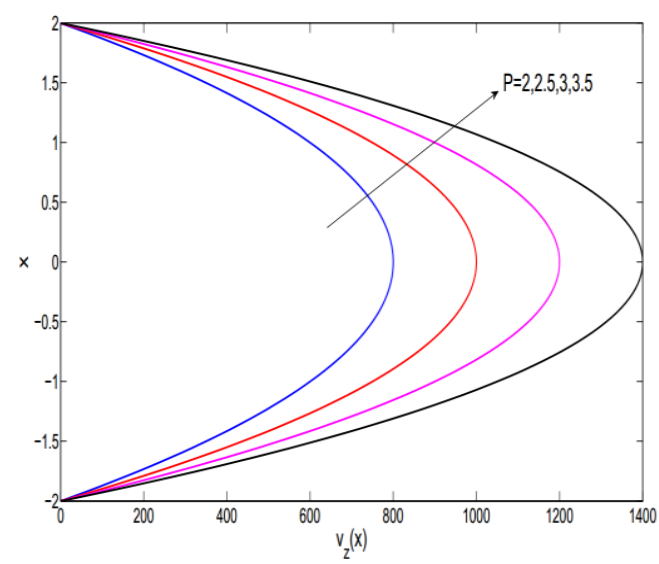

Figure 2: $v_{z}(x)$ versus $x$ (no body couple).

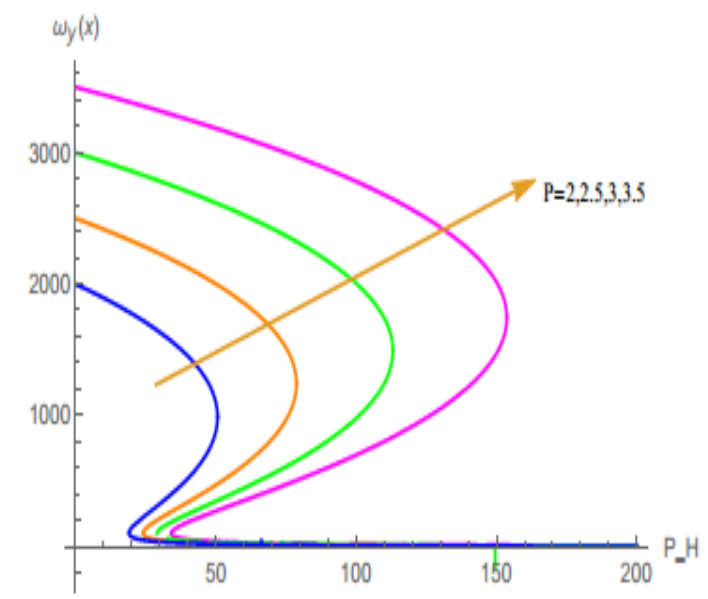

Figure 3: $P_{H}$ versus $\omega_{y}(x)$ for various values of $P$ (with body couple).

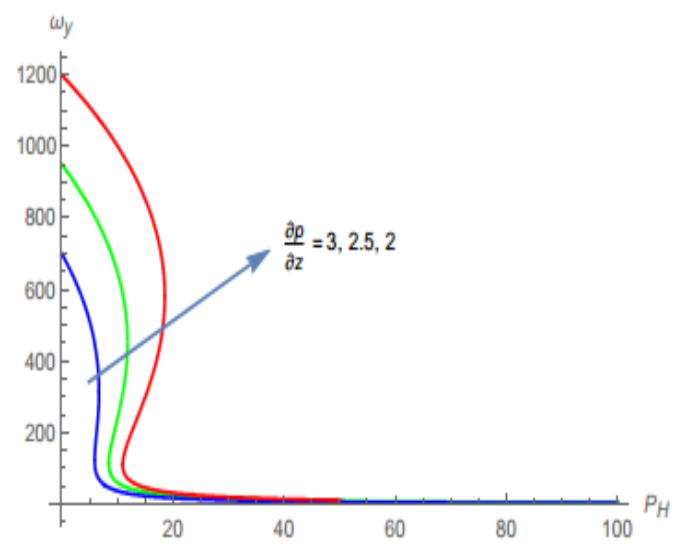


Figure 4: $P_{H}$ versus $\omega_{y}(x)$ for various values of $\frac{\partial p}{\partial z}$ when $\theta=\frac{\pi}{6}$ (with body couple).

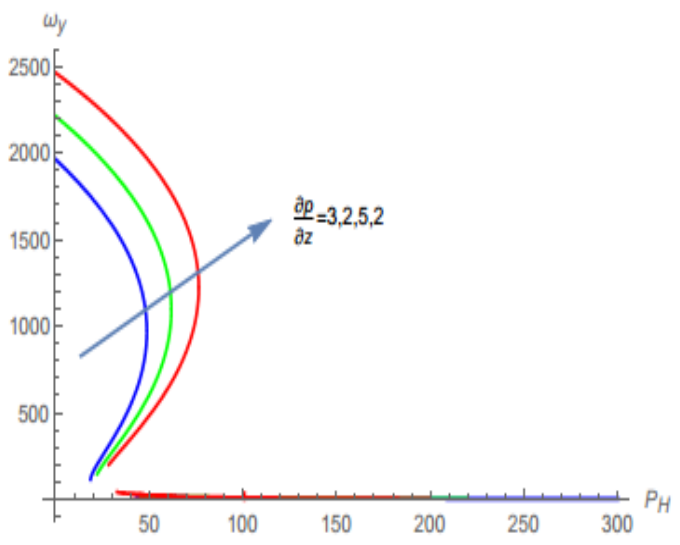

Figure 5: $P_{H}$ versus $\omega_{y}(x)$ for various values of $\frac{\partial p}{\partial z}$ when $\theta=\frac{\pi}{4}$ (with body couple).

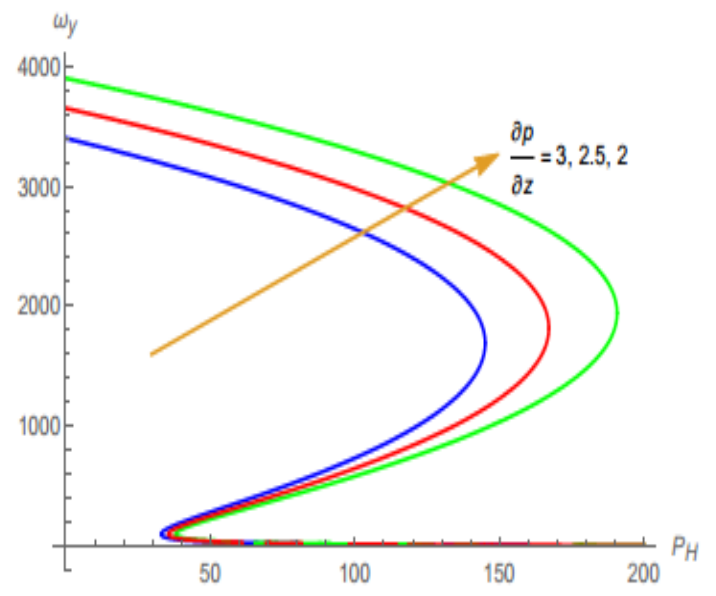

Figure 6: $P_{H}$ versus $\omega_{y}(x)$ for various values of $\frac{\partial p}{\partial z}$ when $\theta=\frac{\pi}{2}$ (with body couple).

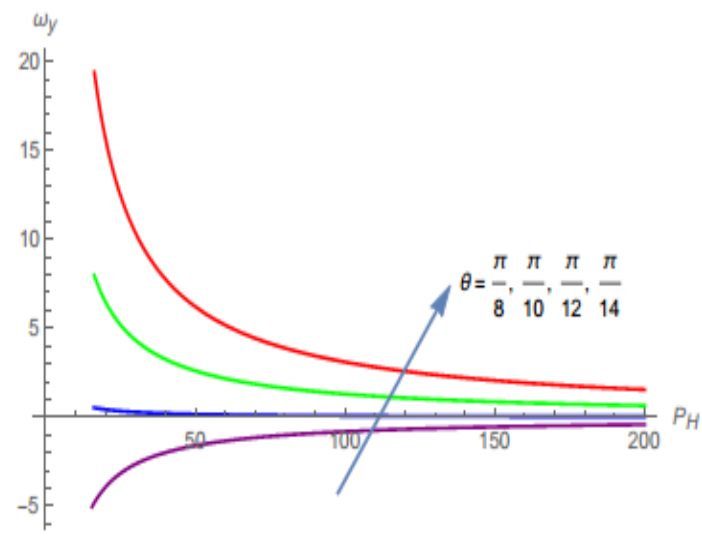

Figure 7: $P_{H}$ versus $\omega_{y}(x)$ for various values of $\theta$ when $\frac{\partial p}{\partial z}=2.5$ (with body couple). 


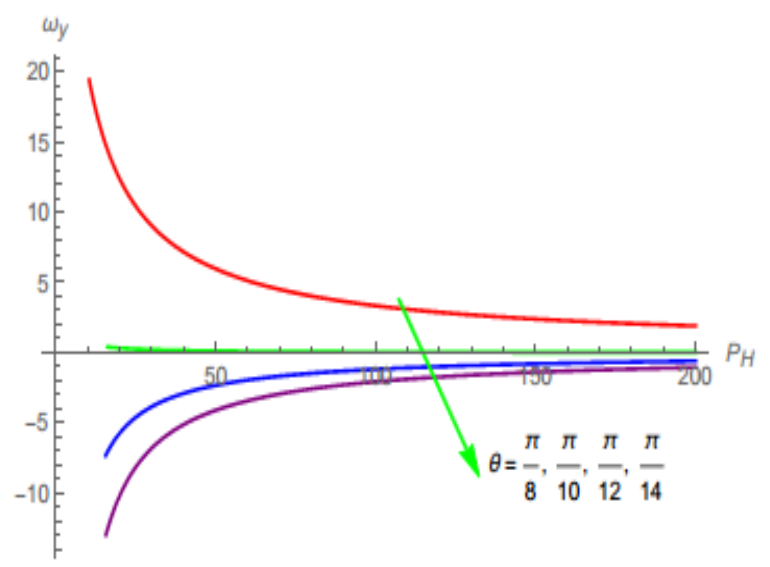

Figure 8: $P_{H}$ versus $\omega_{y}(x)$ for various values of $\theta$ when $\frac{\partial p}{\partial z}=3$ (with body couple).

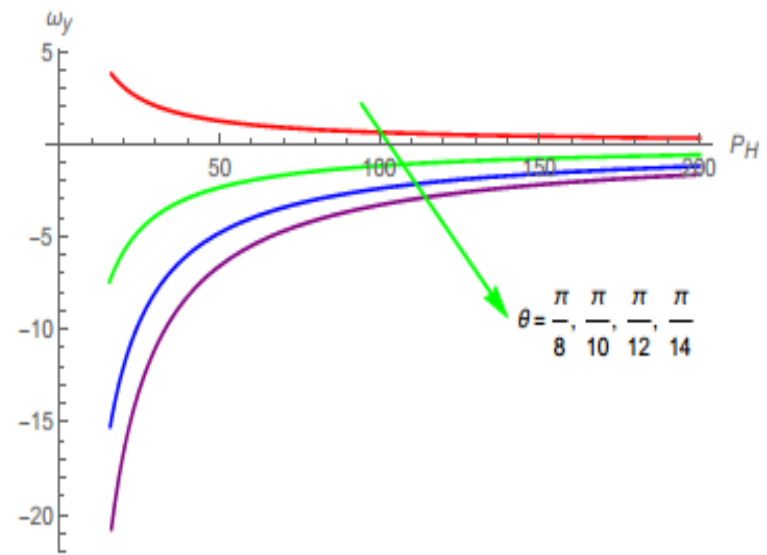

Figure 9: $P_{H}$ versus $\omega_{y}(x)$ for various values of $\theta$ when $\frac{\partial p}{\partial z}=3.5$ (with body couple).

\section{REFERENCES}

[1] S. Odenbach, Colloidal Magnetic fluids, Springer-Verlag Berlin Heidelberg, 2009.

[2] H. Yamaguchi, Engineering fluid Mechanics, Springer,2010.

[3] R.E. Rosensweig, Ferrohydrodynamics, Dover, 2014.

[4] V. G. Bashtovoy, B. M. Berkowsky, A. N. Vislovich, Introduction to Thermomechanics of Magnetic Fluids, Springer, Berlin, 1988.

[5] E. Blums, A. Cebers, M.M. Maiorov, Magnetic Fluids, W. de Gruyter, Berlin, New York, 1997.

[6] G. I. Taylor, Stability of a viscous liquid contained between two rotating cylinders, Philos. Trans. R. Soc. London A 223(1923) $289-343$.

[7] S. Chandrasekhar, Hydrodynamic and Hydromagnetic Stability, Oxford University Press, Oxford, 1966.

[8] P. Drazin, W. Reid, Hydrodynamic Stability, Cambridge University Press, Cambridge, 1981.

[9] E.L. Koschmieder, Benard Cells and Taylor Vortices, Cambridge University Press, Cambridge, 1993.

[10] N. Vislovich, V. A. Novikov, A.K. Sinitsyn, Influence of a magnetic field on the Taylor instability in magnetic fluids, J. Appl. Mech. Tech. Phys. 27 (1986) $72-78$.

[11] M. Niklas, H.M. Krumbhaar, M.H. Lucke, Taylor vortex flow of ferrofluids in the presence of general magnetic fields, J. Magn. Magn. Mater. 81 (1989) 29-38.

[12] P.J. Stiles, M. Kagan, The influence of particle diffusion on the Couette-Taylor instability of a radially magnetized ferroflud, J. Colloid Interf. Sci. 179 (1996) 628-630.

[13] S. Odenbach, H. Gilly, Taylor vortex flow of magnetic fluids under the influence of an azimuthal magnetic field, J. Magn. Magn. Mater. 152(1996) 123128.

[14] M.H. Chang, C.K. Chen, H.C. Weng, Stability of ferrofluid flow between concentric rotating cylinders with an axial magnetic field, Int. J. Eng. Sci. 41 (2003) 103-121.

[15] D.L. Harris, W.H. Reid, On the stability of viscous flow between rotating cylinders, J. Fluid Mech. 20 (part 1) (1964) 95-101.

[16] B.U. Felderhof, Magnetoviscosity and relaxation in ferrofluids, Phys. Rev. E 62 (3) (2000) 3848-3854.

[17] J. P. McTague, J. Chem. Phys. 51, 133(1969).

[18] J. C. Bacri, R. Perzynski, M.I. Shliomis, and G.I. Burde, Phys. Rev. Lett. 75, 2128(1995). 
[19] A. Zeuner, R. Richter, and I. Rehberg, Phys. Rev. E 58, 6287 (1998).

[20] S. Kamiyama, Magnetic Fluids and Applications Handbook, edited by B. Berkovski (Begell House, New york, 1996), p. $475-487$.

[21] M.A. Marsenyuk, Y.L. Raikher, and M.I. Shliomis, Zh. Eksp. Teor. Fiz. 65, 834 (1973) [Sov. Phys. JETP 38, 413 (1974)].

[22] M. Zahn and L.L. Pioch, Ind. J. Eng. Mater. Sci 5, 400 (1998).

[23] F. Gazeau, C. Bharavian, J.C. Bacri, R. Perzynski, and M.I. Shliomis, Energy conversion in ferrofluids: Magnetic nanoparticles as motors or generators, Phys. Rev. E 56, 614 (1997).

[24] K.R. Schumacher, I. Sellien, G.S. Knoke, T. Cader and B.A. Finlayson, Experiment and simulation of laminar and turbulent ferrofluid pipe flow in an oscillating magnetic field, Phy. Rev. 67(026308) (2003) 01-11.

[25] A.P. Krekhov, M.I. Shliomis, S. Kamiyama, Ferrofluid pipe flow in an oscillating magnetic field, Phys. Fluids 17(3) (033105) (2005) 01-08.

[26] J. Singh, R. Bajaj, Couette flow in ferrofluids with magnetic field, J. Magn. Magn. Mater. 294 (2005) 53-62.

[27] X. He, S. Elborai, D. Kim, S.H. Lee, M. Zahn, Effective magnetoviscosity of planar-Couette magnetic fluid flow, J. App. Phys. 97 (2005) 10 Q302 1-3. 